\title{
Three-dimensional radiative transfer effects of clouds in the microwave spectral range
}

\author{
Quanhua Liu, Clemens Simmer, and Eberhard Ruprecht \\ Institut fur Meereskunde, University of Kiel, Kiel, Germany
}

\begin{abstract}
A three-dimensional Monte Carlo transfer model for polarized radiation is developed and used to study three-dimensional (3-D) effects of raining clouds on the microwave brightness temperature. The backward method is combined with the forward method to treat polarization correctly within the cloud. In comparison with horizontally homogeneous clouds, two effects are observed: First, brightness temperatures from clouds are reduced in the 3-D case due to net leakage of radiation from the sidewalls of the cloud. Second, radiation which is emitted by the warm cloud and then reflected from the water surface increases the brightness temperatures of the cloud-free areas in the vicinity of the cloud. Both effects compete with each other, leading to either lower or higher overall brightness temperatures, depending on the geometry of the cloud, the satellite viewing angle, the coverage, and the position of the cloud within the field of view (FOV) of the satellite. At $37 \mathrm{GHz}$, for example, up to $10 \mathrm{~K}$ differences can occur for a cloud of $50 \%$ coverage. Finite homogeneous raining clouds matching the size of the FOV of the satellite show a similar relationship between rain rates and brightness temperatures (TB) as horizontally infinite clouds. Namely, an increase of TB with increasing rain rates at low rain rates, due to emission effects, is followed by a decrease due to temperature and scattering effects. For small horizontal cloud diameter, however, the 3-D brightness temperatures may show a second maximum due to the decrease of the leakage effect with increasing rain rates. At nadir, 3-D brightness temperatures are always lower than the 1-D values with differences up to $20 \mathrm{~K}$ for a cloud of $5-\mathrm{km}$ vertical extent and a base of $1 \times 1$ $\mathrm{km}$. To quantify the 3-D effects for more realistic cloud structures, we used results of a three-dimensional dynamic cloud model as input for the radiative transfer codes. The same 3-D effects are obtained, but the differences between 1-D and 3-D modeling are smaller. In general, most of the differences between the 1-D and 3-D results for off-nadir view angles are pure geometry effects, which can be accounted for in part by a modified 1-D model.
\end{abstract}

\section{Introduction}

Three-dimensional (3-D) radiative transfer effects of clouds are important for estimating precipitation from radiometric observations from satellites and airplanes. The effects depend on the size and the microphysics of the cloud. Owing to these effects, quantitative rain rate determination with microwave radiometry is hampered by the ambiguities of the signal caused by the highly variable vertical profiles of the hydrometeors. This problem has been accounted for in part by using the results of three-dimensional cloud models as a database to restrict the infinite variety of hydrometeor profiles to a small number of realistic ones [Adler et al., 1991]. The radiative transfer calculations, however, have been made with onedimensional transfer codes. Several authors have studied the differences between 3-D and 1-D radiative transfer models. By using a "two orthogonal polarization scheme" to estimate rain rates over water, as described by Spencer [1986], Kummerow and Weinman [1988] demonstrated that the horizontally homogeneous cloud models tend to underestimate rain rates at $50^{\circ}$ viewing angle. They have also shown that calculated brightness temperatures at $50^{\circ}$ viewing angle for moderate to heavy Copyright 1996 by the American Geophysical Union.

Paper number 95JD03421.

0148-0227/96/95JD-03421\$05.00 rain from a finite cloud model are larger than those calculated from a horizontally homogeneous cloud model. However, $\mathrm{Ha}$ ferman et al. [1993] found that the calculated brightness temperatures at nadir from a finite cloud model are smaller than those calculated from a horizontally homogeneous cloud model for all rain rates. In this study a 3-D microwave radiative transfer model is developed based on the backward-forward Monte Carlo method. A comparison is carried out between 1-D and 3-D models. The different results obtained with the other 3-D models, as mentioned above, can be explained by our study.

In the following sections we discuss different approaches in modeling radiative transfer for three-dimensional geometries. In contrast to other similar approaches, our model is able to treat polarization-dependent scattering within clouds correctly. Special attention is paid in section 2 to the effect the model volume size has on the results. In section 3 we test the model by comparing the results with other models. In section 4 , results are shown and discussed for homogeneous box-type clouds and 3-D geometries and profiles taken from a dynamic cloud model.

\section{Methodology}

Various radiative transfer models for horizontally and vertically inhomogeneous atmospheres (3-D models) have been 
developed. The microwave model of Weinman and Davies [1978] for vertically homogeneous problems is based on the eigenvalue method with the first moment of the phase function. Kummerow and Weinman [1988] extended this method to vertically inhomogeneous cases. Stephens [1988] provided a general 3-D radiative transfer model based on the Fourier transform method. A 3-D discrete ordinates model is described by Girens [1993]. Recently, Haferman et al. [1993] studied raining clouds using the discrete ordinates solution procedure, which provides full solution at all grid points and at all directions. McKee and Cox [1974] used the Monte Carlo method (MCM) to study the scattering of visible radiation by finite clouds. Schmetz [1984] uses the MCM to study radiative properties of broken cloud fields. Since we are interested in satellite measurements, we used our Monte Carlo model to calculate the brightness temperature at satellite altitude. For this purpose we applied Mie phase function for a box-type cloud model and Rayleigh phase function for clouds from the dynamic cloud model. The main problems, using the most versatile Monte Carlo radiative transfer models, are the limit of present computer capacity and the treatment of boundaries.

Generally, there are two alternative procedures for the application of the Monte Carlo method: one is the backward method and the other is the forward method. In the forward method the thermal sources within the atmosphere-earth system are simulated by emitting photons from small cells in all directions. Normally, only very few of the emitted photons reach the region of interest (e.g., the radiometer); therefore this method requires a large amount of photons. Thus the method is very time consuming, and its uncertainty is about 2 $\mathrm{K}$ for nonpolarized brightness temperatures [Weinman and Davies, 1978]. The backward method retraces the photons from the receiver (e.g., satellite radiometer) to their source, where they have been emitted. This method is very time efficient, because only those photons are considered which actually reach the receiver. The backward method has also been applied to the microwave spectral region by Roberti and Kummerow [1995].

We combine backward and forward methods. The backward method is used to determine those photons which contribute to the signal at the radiometer. Then the forward method is used only for these photons to determine their contribution and polarization state at the radiometer. This combination is almost as fast as a pure backward method but treats the polarization within clouds correctly. For our MCM four random numbers $R_{1}$ are required, which represent the stochastic processes of the photons (for our method we should rather say energy particles), and they are generated by the computer. The first random number is

$$
R_{1}=1-\exp \left[-\int_{0}^{s} \beta(x, y, z) d s\right]
$$

where $\beta$ is the volume extinction coefficient, $d s$ is differential distance, and the integral limit $s$ is the distance the photon will travel until it encounters an extinction event.

Whether the photon is scattered or absorbed during an extinction event is determined by the second random number

$$
\begin{array}{ll}
\text { Scattered } & R_{2}-\bar{\omega}(x, y, z) \leq 0, \\
\text { Absorbed } & R_{2}-\bar{\omega}(x, y, z)>0,
\end{array}
$$

where $\bar{\omega}$ is the local single scattering albedo.
The scattering angle $\Theta$ of the photon is computed from the third random number

$$
R_{3}=2 \pi \int_{0}^{\Theta} P(\theta) d \cos (\theta)
$$

where $P(\theta)$ is the phase function for total intensity.

The second angle $\gamma$ is a rotation angle about the direction of propagation prior to the collision. The angle $\gamma$ is chosen randomly between 0 and $2 \pi$ by the fourth random number

$$
\gamma=2 \pi R_{4}
$$

With the four random numbers $R_{t}$ the status of the photon is determined. $R_{1}$ is used to calculate the traveled distance of the photon. $R_{2}$ determines whether the photon is absorbed or scattered. $R_{3}$ and $R_{4}$ determine via the angles $\Theta$ and $\gamma$ the new travel direction of the scattered photon. The photon is traced from the radiometer backward until $R_{2}$ indicates an absorption event. Several scattering events may have occurred to the photon before absorption. When an absorption event occurs, we switch to the forward mode and retrace the photon path. We assume that the unpolarized emission from each local source consists of two photons; one is treated in the forward calculation as vertically polarized and the other as horizontally polarized. The Stokes vector of the two photons after $k$ scattering events is determined by forward calculation using

$$
\begin{gathered}
{\left[\begin{array}{c}
I_{v} \\
I_{h} \\
U \\
V
\end{array}\right]=S_{k} \cdots S_{2} S_{1}\left[\begin{array}{c}
I_{v 0} \\
I_{h 0} \\
0 \\
0
\end{array}\right]} \\
I_{v 0}=I_{h 0}=I_{0}
\end{gathered}
$$

where $I_{0}$ is the energy of the local source, $I_{\mathrm{v}}$ and $I_{\mathrm{h}}$ are the energies at vertical and horizontal polarization after $k$ scattering events, respectively. The renormalized scattering matrix $S_{v}$, which represents the $i$ th scattering process, is calculated from the rotated phase matrix (the subscript $i$ is dropped in the following)

$$
S=\frac{1}{P}\left[\begin{array}{llll}
P_{11} & P_{12} & P_{13} & P_{14} \\
P_{21} & P_{22} & P_{23} & P_{24} \\
P_{31} & P_{32} & P_{33} & P_{34} \\
P_{41} & P_{42} & P_{43} & P_{44}
\end{array}\right]
$$

where $P=\left(P_{11}+P_{12}+P_{21}+P_{22}\right) / 2$ is the phase function for the total intensity.

The elements $P_{\imath j}$ of the rotated phase matrix are calculated according to Tsang et al., [1985]:

$$
L\left(i_{2}-\pi\right) \bar{P}(\Theta) L\left(i_{1}\right)=\left[\begin{array}{llll}
P_{11} & P_{12} & P_{13} & P_{14} \\
P_{21} & P_{22} & P_{23} & P_{24} \\
P_{31} & P_{32} & P_{33} & P_{34} \\
P_{41} & P_{42} & P_{43} & P_{44}
\end{array}\right]
$$

where $L$ is the rotation matrix. The rotation angles $i_{1}$ and $i_{2}$ depend on the incoming and outgoing directions. $\bar{P}$ is the scattering matrix, which depends only on the local scattering angle and the optical properties of the medium.

Calculations are carried out for a rectangular volume of 800 $\mathrm{km} \times 800 \mathrm{~km}$ in the horizontal and $40 \mathrm{~km}$ in the vertical direction. The cloud is placed in the center of the volume. Unrealistic flux exchange through the lateral boundaries causes an error of less than $0.2 \mathrm{~K}$. The space above $40 \mathrm{~km}$ is 


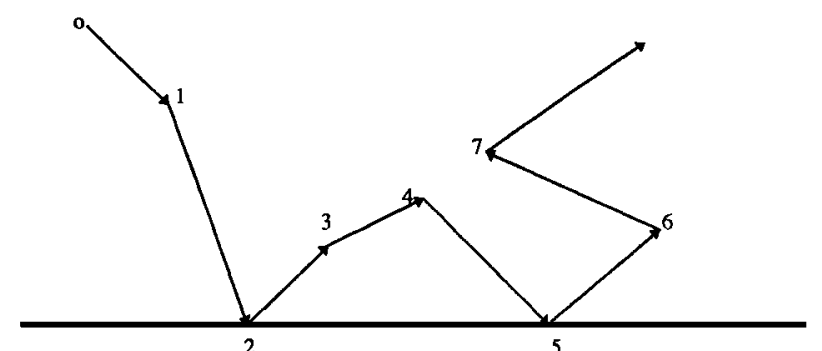

Figure 1. Schematic description of a sequence of scattering and reflection events of a photon in the Monte Carlo method.

considered vacuum. The lower boundary is modeled as an ocean surface. Its reflectances for vertically and horizontally polarized radiation are calculated after Wisler and Hollinger [1977].

In order to illustrate the calculation steps, an example is given in Figure 1. Two photons with equal energy which are emitted from position 0 undertake the following events: scattering at point 1 , reflection at point 2 , scattering at points 3 and 4 , reflection at point 5 , and scattering at points 6 and 7 . For this case the energy reaching the radiometer is

$$
\begin{gathered}
{\left[\begin{array}{c}
I_{\mathrm{v}} \\
I_{\mathrm{h}} \\
U \\
V
\end{array}\right]=S_{7} S_{6} r_{5} S_{4} S_{3} r_{2} S_{1}\left[\begin{array}{c}
I_{\mathrm{v} 1} \\
I_{\mathrm{h} 0} \\
0 \\
0
\end{array}\right]+S_{7} S_{6} r_{5} S_{4} S_{3}\left(E-r_{2}\right)\left[\begin{array}{c}
I_{\mathrm{v} 2} \\
I_{\mathrm{h} 2} \\
0 \\
0
\end{array}\right]} \\
+S_{7} S_{6}\left(E-r_{5}\right)\left[\begin{array}{c}
I_{\mathrm{v} 5} \\
I_{\mathrm{h} 5} \\
0 \\
0
\end{array}\right] \\
I_{\mathrm{v} 0}=I_{\mathrm{h} 10}=I_{0} \\
I_{\mathrm{v} 2}=I_{\mathrm{h} 2}=I_{2} \\
I_{\mathrm{v} 5}=I_{\mathrm{h} 5}=I_{5}
\end{gathered}
$$

$I_{0}, I_{2}$, and $I_{5}$ are local sources with energies proportional to the local temperature. $E$ is the unit matrix. The scattering matrix $S$ is given by (6) and the reflection matrix $r$ for a calm water surface is defined by

$$
r=\left[\begin{array}{cccc}
\left|R_{\mathrm{v}}\right|^{2} & 0 & 0 & 0 \\
0 & \left|R_{\mathrm{h}}\right|^{2} & 0 & 0 \\
0 & 0 & \operatorname{Re}\left(R_{\mathrm{v}} R_{\mathrm{h}}^{*}\right) & -\operatorname{Im}\left(R_{\mathrm{v}} R_{\mathrm{h}}^{*}\right) \\
0 & 0 & \operatorname{Im}\left(R_{\mathrm{h}} R_{\mathrm{v}}^{*}\right) & \operatorname{Re}\left(R_{\mathrm{h}} R_{\mathrm{v}}^{*}\right)
\end{array}\right]
$$

where $R_{\mathrm{v}}$ and $R_{\mathrm{h}}$ are the local Fresnel coefficients. $R_{\mathrm{v}}^{*}$ and $R_{\mathrm{h}}^{*}$ are the complex conjugates of $R_{\mathrm{v}}$ and $R_{\mathrm{h}}$. The first term of the right-hand side of (8) is the contribution from point 0 ; the second and third terms are contributions of the emission by the ocean surface to the signal at the radiometer.

In 3-D modeling of radiative transfer, unrealistic exchange of radiation through the lateral boundaries takes place. In order to analyze these effects, we placed a box-type rain cloud with fixed dimensions $(1 \times 1 \mathrm{~km}$ horizontal diameter, $5 \mathrm{~km}$ vertical extension) in the center of the model area. Then we extended step-wise the horizontal dimensions of the total model area, that means, the cloud-free environment around the cloud increases. The model area is surrounded by vacuum. The cloud has a horizontally and vertically homogeneous distribution of rain water with a rain rate of $5 \mathrm{~mm} \mathrm{~h}^{-1}$. The
Marshall-Palmer distribution is used to describe the drop-size spectrum. The temperature within and outside of the cloud is $290 \mathrm{~K}$ at the surface with a linear decrease up to the freezing level at $5 \mathrm{~km}$. The absorption coefficients of the atmospheric gases are taken from Liebe [1985]; the radiative properties of the hydrometeors of the cloud are computed from Mie theory. Wind speed at the ocean surface is set to $5 \mathrm{~m} \mathrm{~s}^{-1}$.

Increasing the size of the cloud-free environment results in an increase of the nadir cloud-top brightness temperature (Table 1). The observed differences result from a decreasing net loss of radiation through the lateral boundaries of the cloud. These differences depend on the ratio of the area of the cloud sidewalls to the top and bottom area of the cloud. When the size of the cloud free environment is extended to several hundred kilometers, the differences are reduced to about 0.1 K. Therefore results presented in the sections 3 and 4 are calculated with a model area of $800 \times 800 \mathrm{~km}$.

\section{Comparison With Other Radiative Transfer Models}

In order to test our model, it was intercompared with other models. For the first comparison we used our 1-D model based on the matrix operator method (MOM) [Liu, 1990; Liu et al., 1991]. The aim of this test was to check whether the polarization-dependent scattering is correctly treated in our 3-D model. For this purpose we assumed horizontally homogeneous and infinite clouds. The parameterizations of the singlescattering albedo $\bar{\omega}$ and optical depth $\delta$ with rainfall rates (RR) are taken from Weinman and Guetter [1977]. The top of the raining cloud is at $4.57 \mathrm{~km}$ with a temperature of $258 \mathrm{~K}$. The base temperature is $288 \mathrm{~K}$. The results of the two models agree within $0.2 \mathrm{~K}$ (Table 2). This implies that the method to treat polarization in the MCM is correct.

Second, a comparison of our two models (3-D MCM, 1-D MOM) was performed with results of the 3-D model and the analytical method by Weinman and Davies [1978] applied to a finite cubic cloud (Table 3 ). The horizontal and vertical scales are determined by the optical depth in $x, y$, and $z$ directions. The temperature of the homogeneous cloud is $275 \mathrm{~K}$. Outside of the cubic cloud is vacuum with no fluxes from the outside into the cloud. Results calculated with the four models agree in general. Except for the horizontally infinite cloud the brightness temperatures from the models of Weinman and Davies [1978] are always lower. Differences are below $1 \mathrm{~K}$ for very small horizontal optical depth and increase somewhat with

Table 1. Effects of the Size of the Cloud-Free Environment Around a Rain Cloud on Brightness Temperature at Nadir

\begin{tabular}{cc}
$\begin{array}{c}\text { Environment } \\
\text { Around Cloud, } \\
\mathrm{km}\end{array}$ & $\begin{array}{c}\text { Brightness } \\
\text { Temperature, } \\
\mathbf{K}\end{array}$ \\
\hline 0 & 210.2 \\
1 & 214.8 \\
10 & 228.8 \\
50 & 233.8 \\
200 & 234.6 \\
500 & 234.7
\end{tabular}

The rain cloud is $1 \times 1 \mathrm{~km}$ horizontal size with a height of $5 \mathrm{~km}$. The environment is cloud-free with otherwise the same properties as in the cloud. The rain rate is $5 \mathrm{~mm} \mathrm{~h}^{-1}$. 
Table 2. Comparison of Brightness Temperatures Calculated With Monte Carlo Method (first row) and Matrix Operator Method (second row) for Horizontally Homogeneous and Infinite Clouds

\begin{tabular}{|c|c|c|c|c|c|}
\hline \multirow{2}{*}{$\begin{array}{l}\mathrm{RR}, \\
\mathrm{mm} \mathrm{h}^{-1}\end{array}$} & \multirow[b]{2}{*}{$\bar{\omega} / \delta$} & \multicolumn{2}{|c|}{$\theta=26$} & \multicolumn{2}{|c|}{$\theta=49$} \\
\hline & & Horizontal & Vertical & Horizontal & Vertical \\
\hline \multirow[t]{2}{*}{1} & $0.20 / 0.37$ & 196.9 & 207.1 & 201.9 & 232.8 \\
\hline & & 196.9 & 207.1 & 201.9 & 232.7 \\
\hline \multirow[t]{2}{*}{2} & $0.23 / 0.71$ & 227.9 & 233.5 & 234.8 & 248.5 \\
\hline & & 227.9 & 233.5 & 234.6 & 248.4 \\
\hline \multirow[t]{2}{*}{4} & $0.27 / 1.33$ & 247.4 & 249.5 & 247.8 & 252.3 \\
\hline & & 247.3 & 249.5 & 247.7 & 252.4 \\
\hline \multirow[t]{2}{*}{8} & $0.33 / 2.59$ & 247.9 & 249.0 & 243.0 & 245.6 \\
\hline & & 247.9 & 248.8 & 243.0 & 245.5 \\
\hline \multirow[t]{2}{*}{16} & $0.37 / 5.11$ & 241.4 & 242.5 & 236.3 & 239.0 \\
\hline & & 241.4 & 242.3 & 236.2 & 238.9 \\
\hline \multirow[t]{2}{*}{32} & $0.40 / 10.2$ & 236.2 & 237.4 & 231.3 & 234.5 \\
\hline & & 236.3 & 237.2 & 231.4 & 234.3 \\
\hline
\end{tabular}

$\mathrm{RR}$ is the rainfall rate, $\theta$ is the viewing angle, $\bar{\omega}$ is the singlescattering albedo, and $\delta$ is the optical thickness.

vertical optical depth. The largest difference $(6.9 \mathrm{~K})$ is found between our MCM and the analytical method for the horizontally infinite cloud at moderate vertical optical depths. In general, the agreement of both our models with Weinman and Davies' MCM is better than with their analytical method. The results of Table 3 make us confident about the good quality of our MCM code.

Table 3. Mean Brightness Temperature at Viewing Angle $\theta=50^{\circ}$ for an Isothermal Cloud Computed by the Monte Carlo Method (MCM) and the Analytical Method of Weinman and Davies [1978]

\begin{tabular}{|c|c|c|c|c|c|c|}
\hline \multirow[b]{2}{*}{$\delta z$} & \multirow[b]{2}{*}{ Method } & \multicolumn{5}{|c|}{$\delta x=\delta y$} \\
\hline & & 0.3 & 1 & 3 & 10 & $\infty$ \\
\hline \multirow[t]{4}{*}{0.3} & A & $\begin{array}{c}21.6 \\
(0.2)\end{array}$ & $\begin{array}{l}34.9 \\
(0.6)\end{array}$ & $\begin{array}{l}44.0 \\
(0.6)\end{array}$ & $\begin{array}{l}48.7 \\
(0.7)\end{array}$ & $\begin{array}{c}52.3 \\
(0.3)\end{array}$ \\
\hline & B & 21.7 & 34.7 & 42.6 & 46.3 & 48.1 \\
\hline & C & 22.0 & 35.6 & 44.5 & 49.0 & 51.3 \\
\hline & D & & & & & 51.4 \\
\hline \multirow[t]{3}{*}{1} & A & $\begin{array}{l}31.1 \\
(0.5)\end{array}$ & $\begin{array}{l}63.5 \\
(1.0)\end{array}$ & $\begin{array}{l}94.6 \\
(1.4)\end{array}$ & $\begin{array}{l}119 \\
(2.0)\end{array}$ & $\begin{array}{r}136.7 \\
(0.7)\end{array}$ \\
\hline & B & 31.3 & 62.0 & 92.5 & 116 & 129.7 \\
\hline & $\begin{array}{l}\text { C } \\
\text { D }\end{array}$ & 31.9 & 63.8 & 96.0 & 121.1 & $\begin{array}{l}136.6 \\
136.9\end{array}$ \\
\hline \multirow[t]{3}{*}{3} & A & $\begin{array}{c}35.9 \\
(0.6)\end{array}$ & $\begin{array}{l}85 \\
(2.0)\end{array}$ & $\begin{array}{l}131 \\
(2.0)\end{array}$ & $\begin{array}{l}169 \\
(3.0)\end{array}$ & $\begin{array}{c}210 \\
(1.0)\end{array}$ \\
\hline & B & 36.0 & 84 & 130 & 171 & 208 \\
\hline & $\begin{array}{l}\text { C } \\
\text { D }\end{array}$ & 36.8 & 86.6 & 132.9 & 173.3 & $\begin{array}{l}210.9 \\
211.2\end{array}$ \\
\hline \multirow[t]{4}{*}{10} & A & $\begin{array}{c}37.9 \\
(0.6)\end{array}$ & $\begin{array}{l}99 \\
(2.0)\end{array}$ & $\begin{array}{l}158 \\
(2.0)\end{array}$ & $\begin{array}{l}189 \\
(3.0)\end{array}$ & $\begin{array}{r}224.5 \\
(1.5)\end{array}$ \\
\hline & B & 38.0 & 97 & 159 & 191 & 219 \\
\hline & C & 38.9 & 100.5 & 162.6 & 191.9 & 221.9 \\
\hline & D & & & & & 222.1 \\
\hline
\end{tabular}

The value in parentheses is the uncertainty of the MCM given by Weinman and Davies [1978]. The cloud is characterized by asymmetry factor $g=0$, constant temperature $T=275 \mathrm{~K}$, and single-scattering albedo $\bar{\omega}=0.6$. The environment around the cloud is assumed to be vacuum. A, MCM of Weinman and Davies [1978]; B, analytical method from Weinman and Davies [1978]; C, MCM, our model; D, matrix operator method for an infinite cloud; $\delta x=\delta y$, optical depth in horizontal directions $x$ and $y$, respectively and $\delta z$, optical depth in vertical direction $z$.

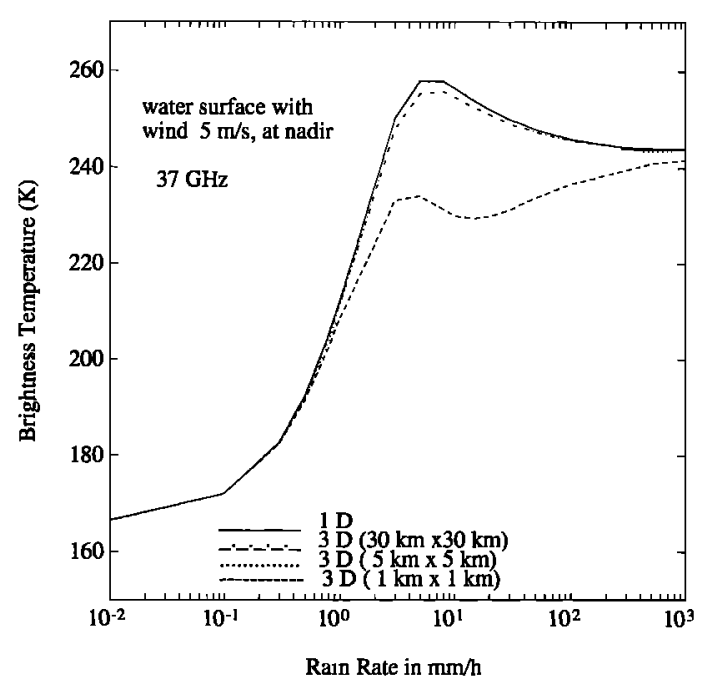

Figure 2a. Relationship between $37 \mathrm{GHz}$ brightness temperature at nadir and rain rates for different horizontal scales of raining clouds (1-D corresponds to a horizontally infinite cloud).

\section{Results}

In order to understand and quantify the effects that the three-dimensional structure of a raining cloud has on the transfer of microwave radiation, we applied our backward-forward method to two different types of cloud models. A box-type cloud model was analyzed to explain the physics of the observed effects. A three-dimensional dynamic cloud model was applied to create more realistic cloud structures, which enable us to quantify the effects expected from real clouds.

\subsection{Box-Type Cloud Model}

It was stated above, that the microwave radiances leaving the cloud top depend on the ratio between the area of the cloud sidewalls and its surface area. This effect is investigated with the box-type cloud model in more detail. We define a box-type cloud model as a finite cloud with horizontally and vertically homogeneous precipitation distribution. The box-type clouds are always $5 \mathrm{~km}$ thick, and the cloud-free environment has the same temperature and water vapor structure as the cloud. In the first study the brightness temperatures (TB), averaged over the cloud-top area, are calculated for a nadir-looking radiometer for different cloud sizes and varying rain rates. For comparison, the results of the 1-D model, that is, horizontally infinite clouds, are also shown. For both frequencies, $37 \mathrm{GHz}$ (Figure 2a) and $19.35 \mathrm{GHz}$ (Figure 2b), TB increase with increasing rain rates, reach a maximum, and decrease for higher rain rates. This general behavior is already known as a result of the 1-D model. The increase of TB is caused by increasing cloud emissivity. The decrease of TB is caused by scattering effects and the upward shift (temperature decrease) of the layer that contributes most to the radiances. The results of both frequencies have similar structures. The maximum of TB is between 3 and $7 \mathrm{~mm} \mathrm{~h}^{-1}$ for the $37 \mathrm{GHz}$ frequency and between 10 and $20 \mathrm{~mm} \mathrm{~h}^{-1}$ for the $19.35 \mathrm{GHz}$ frequency. The differences between the 1-D and the 3-D models are larger for the higher frequencies. Leakage of radiation from the sidewalls of the cloud to the radiatively colder cloud-free environment causes lower TB, when 3-D effects are taken into account. The differences, compared to the horizontally homogeneous cloud, 


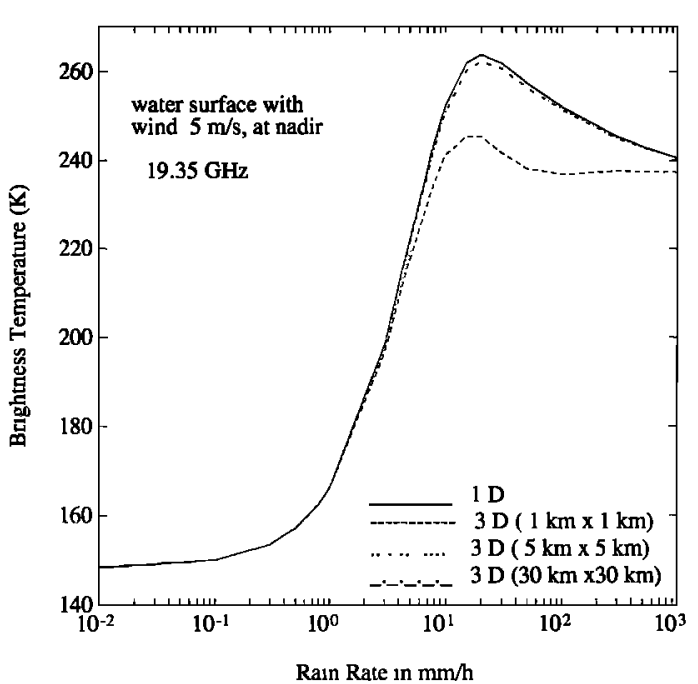

Figure 2b. Same as in Figure 2a but for $19.35 \mathrm{GHz}$.

increase with decreasing cloud diameter, as expected. The differences decrease also with decreasing rain rate because of the diminishing emissivity difference between cloud and environment. For the $1 \times 1-\mathrm{km}$ cloud the reduction of the side leakage effect due to increasing optical depth is larger than the TB reduction caused by scattering and upward shift of the contributing level, leading to another TB increase.

In order to study the effect of cloud coverage within the satellite FOV, a cloud with $5 \mathrm{~mm} \mathrm{~h}^{-1}$ of varying diameter is placed in the center of the FOV. Three different sizes of FOV are investigated: $1 \times 1 \mathrm{~km}, 5 \times 5 \mathrm{~km}$, and $30 \times 30 \mathrm{~km}$ (Figures $3 \mathrm{a}$ and $3 \mathrm{~b}$ ). Calculations were performed for a viewing angle of $53^{\circ}$, to simulate current satellite sensor geometries. The 1-D radiative transfer model leads to a linear relation between cloud cover and TB, independent of the size of the FOV and frequency. This is different for the $3-\mathrm{D}$ model. The relation between cloud cover and TB is nonlinear for all sizes of FOV. For large FOVs the relation is highly nonlinear. TB is always

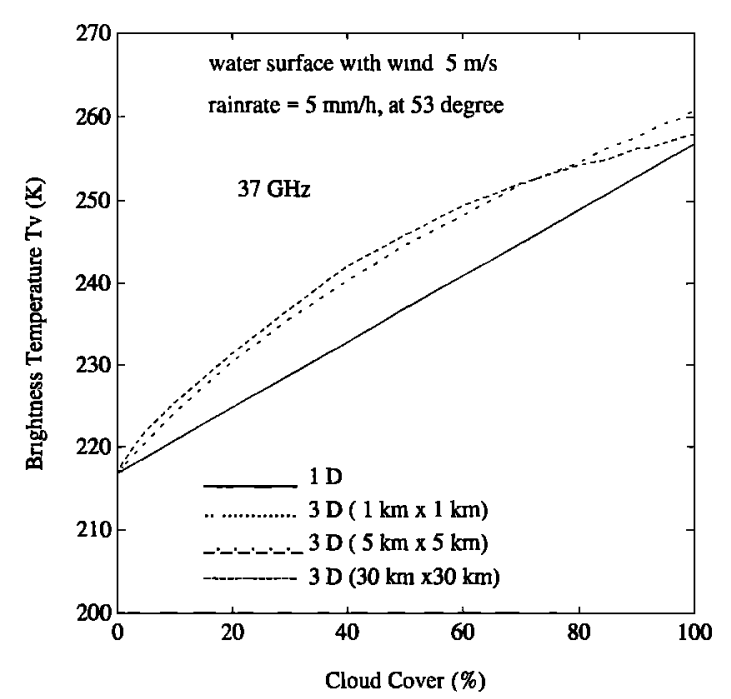

Figure 3a. Relationship between $37 \mathrm{GHz}$ vertically polarized brightness temperature at a viewing angle of $53^{\circ}$ and cloud coverage for different horizontal scale of raining clouds (1-D corresponds to a horizontally infinite cloud).

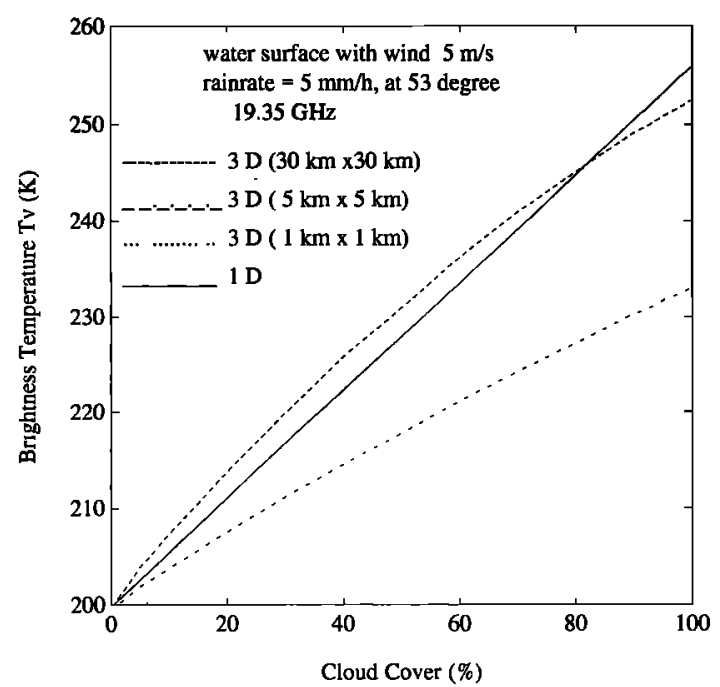

Figure 3b. Same as in Figure 3a but for $19.35 \mathrm{GHz}$.

underestimated by the 1-D model. For small FOVs the 3-D simulations show an almost linear increase with cloud cover, but TBs are always lower than calculated by the 1-D model. The higher 3-D brightness temperatures for large FOVs are caused by the sidewalls, because at a viewing angle of $53^{\circ}$ the radiometer sees both the cloud top and the sidewalls. The brightness temperatures of the sidewalls are higher than those of the cloud tops, due to the decrease of the temperature with height. In addition, the radiometer measures the reflected radiation from the warm sidewalls when viewing the cloud-free ocean surface close to the cloud. Both effects overcompensate for the net leakage effect described above. For smaller clouds the leakage effect dominates the signal. At $19.35 \mathrm{GHz}$ (Figure 3b) the radiation from the warm sidewalls has less energy than at $37 \mathrm{GHz}$ (Figure 3a) due to the lower emissivity of the cloud. Therefore overcompensation for the net leakage effect occurs only for the larger FOVs. Increasing rain rates will increase the range where the effect of the warm sidewalls dominates the signal.

The effect of the warm sidewalls and the reflection at the ocean surface are clearly demonstrated when changing the position of the cloud within the FOV (Figure 4). The largest brightness temperatures are obtained when the cloud is concentrated in the far-off portion of the FOV with respect to the satellite viewing direction. Then the satellite views a maximum of the warm sidewall area and its reflection from the ocean surface (Figure 4c). The lowest brightness temperature occurs when only a small sidewall area, the cloud top, and the cold cloud-free ocean surface without reflection from the cloud are seen by the radiometer (Figure $4 b$ ).

These effects are illustrated best by simulating the measurements of a high-resolution radiometer on an airplane flying over the cloud. For our example, we assume a top height of 5 $\mathrm{km}$ and a diameter of $10 \times 10 \mathrm{~km}$ (Figures $5 \mathrm{a}$ and $5 \mathrm{~b}$ ). The plane passes from west to east (left to right in Figures 5a and $5 \mathrm{~b}$ ), and the radiometer looks foward at an angle of $53^{\circ}$ with a spatial resolution of $1 \times 1 \mathrm{~km}$. At $37 \mathrm{GHz}$ the radiometer receives the first signal from the cloud, the reflection of the upper part of the cloud sidewall, at about $6 \mathrm{~km}$ away from the cloud edge (Figure 5a). The signal increases and reaches its maximum when the warm cloud base enters the FOV. As the plane continues to approach the cloud, the brightness temper- 




$\mathrm{T} 37 \mathrm{v}=246.4 \mathrm{~K}$

a

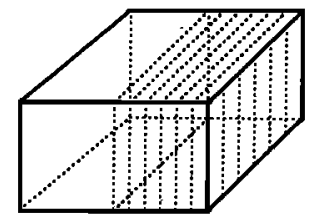

$T 37 v=2642 K$

c

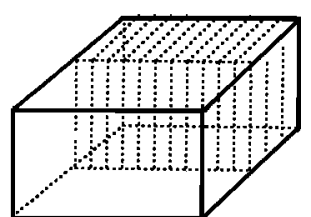

T37v $=237.5 \mathrm{~K}$

b

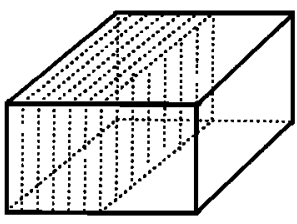

$\mathrm{T} 37 \mathrm{v}=252.0 \mathrm{~K}$

d

Figure 4. Mean $37 \mathrm{GHz}$ vertically polarized brightness temperature for various positions of the cloud (dotted) within the FOV. The radiometer observes the cloud from left to right at $53^{\circ}$. Cloud coverage is fixed at $50 \%$, and the rainfall rate is 5 $\mathrm{mm} \mathrm{h}^{-1}$.

ature decreases because the radiometer scans the sidewall of the cloud upward toward the cold cloud top. The signal levels off when the cloud top enters the FOV and shows a slight increase scanning toward the cloud top center (decreasing leakage effect). The signal drops down again when the radiometer begins to sense the cold ocean surface through the far edge of the cloud. The radiometer hits the cloud-free ocean surface about $6 \mathrm{~km}$ behind the cloud. At $19 \mathrm{GHz}$ the cloud is transparent at all viewing positions (Figure $5 \mathrm{~b}$ ). Therefore the signal is strongly influenced also by emission from and within the cloud, causing a less sharp structure of the brightness temperature and the shift of the maximum toward the cloud center.

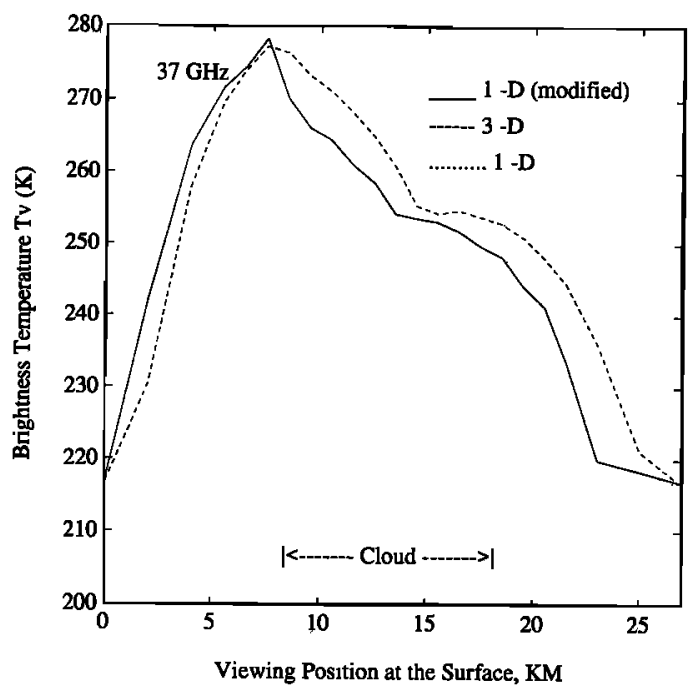

Figure 5a. $37 \mathrm{GHz}$ vertically polarized brightness temperature at different viewing positions. Viewing angle is $53^{\circ}$; the abscissa gives the position at the surface of the line of sight from the satellite toward the ground. The rainfall rate is $5 \mathrm{~mm}$ $\mathrm{h}^{-1}$.

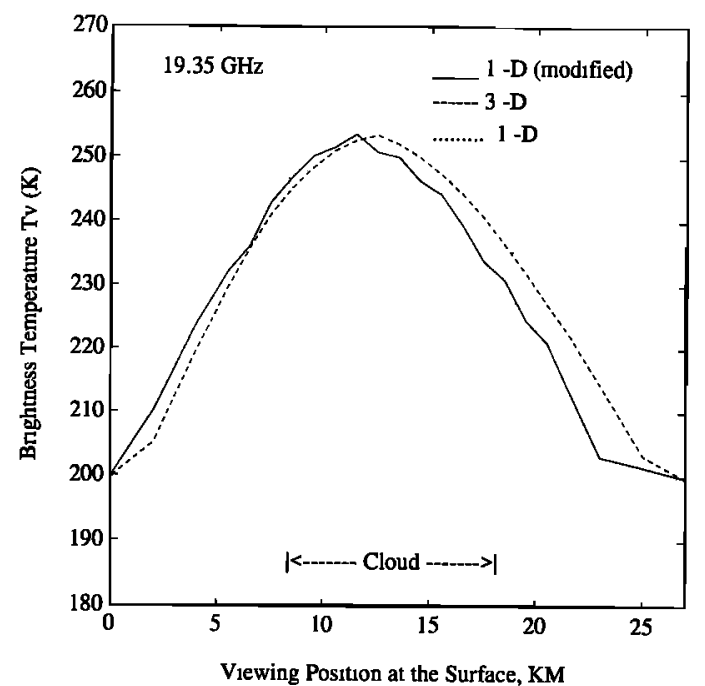

Figure 5b. Same as in Figure 5a but for $19.35 \mathrm{GHz}$.

The large differences between 1-D and 3-D radiative transfer calculations for off-nadir observations are mainly due to the effects of geometry. A modified 1-D model can already account for the geometry effects (Figure 6). For each viewing position we performed two 1-D calculations using clouds of different vertical structure. In this modified model the viewing geometry of the radiometer through the cloud is taken into account (Figure 6). For the calculation of the upward radiation a horizontally homogeneous cloud is assumed. The vertical profile of this hypothetical cloud is given by an intersection of the line of sight from the radiometer to the surface through the real cloud. For the downward radiation, which is reflected at the surface, the hypothetical cloud is defined by the intersection of the specular reflected line of sight through the real cloud. The result of this modified 1-D model is much closer to the 3-D model (Figures 5a and 5b). The remaining differences can be attributed to effects of 3-D radiative transfer alone.

Radiative transfer problem with a finte cloud

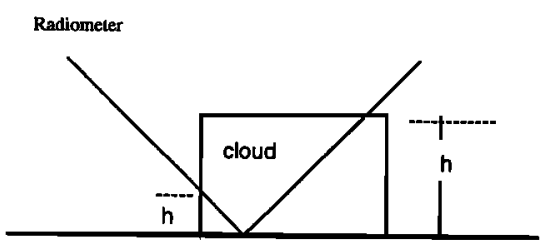

Calculating TB with a modıfied ID radiative transfer model.
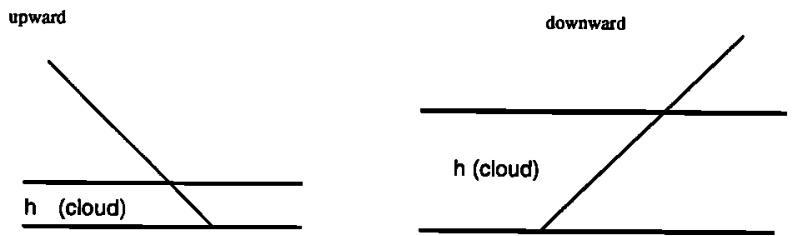

Figure 6. Schematic picture of the modified 1-D radiative transfer model for a finite cloud. 


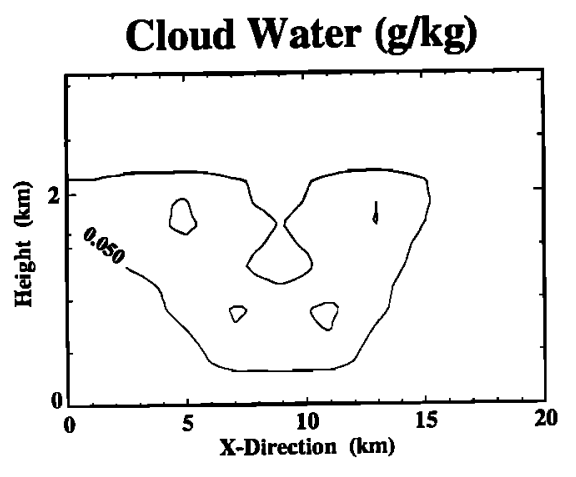

Section al $Y=11 \mathrm{~km}$, Time: 40:00 min Max: 0.11

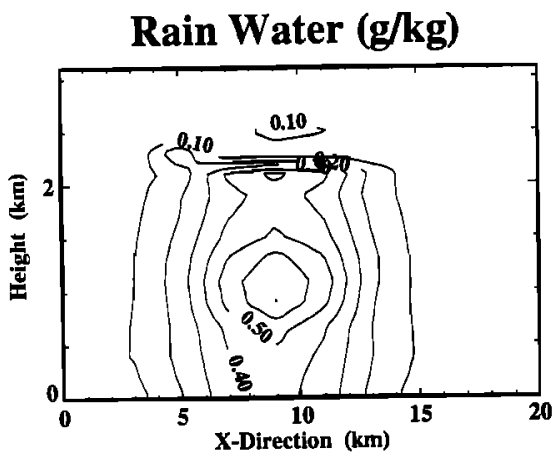

Sectlon at $Y=11 \mathrm{~km}$, Time: 40:00 min Mrx.: 0.70
Total Ice Content $(\mathrm{g} / \mathrm{kg})$

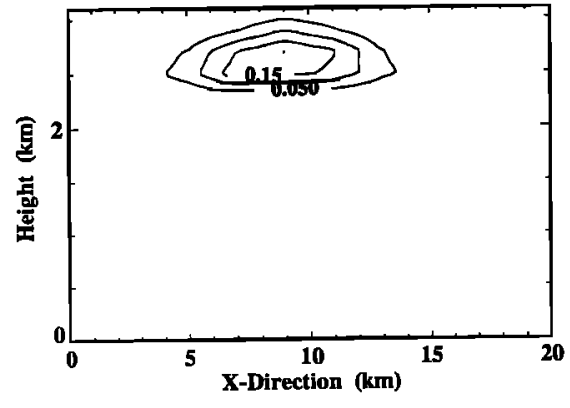

Section at $Y=11 \mathrm{~lm}$, Time: 40:00 min Mex: 0.20

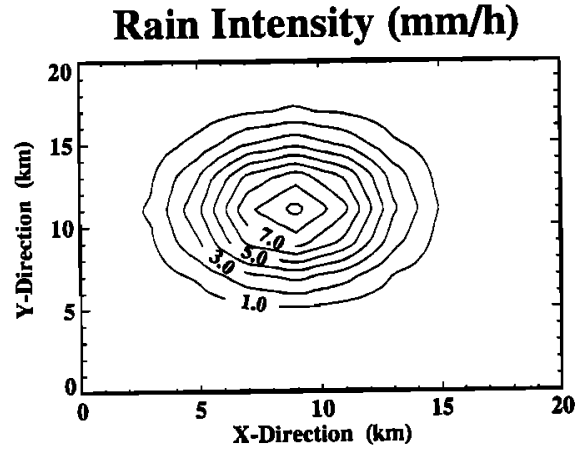

Height: $0.05 \mathrm{~km}$, Time: 40:00 min Max: 9.31

Figure 7. Height cross section through the center of the simulated cloud of cloud water, of rain water, and of ice water content and rain intensity at $50 \mathrm{~m}$ level.
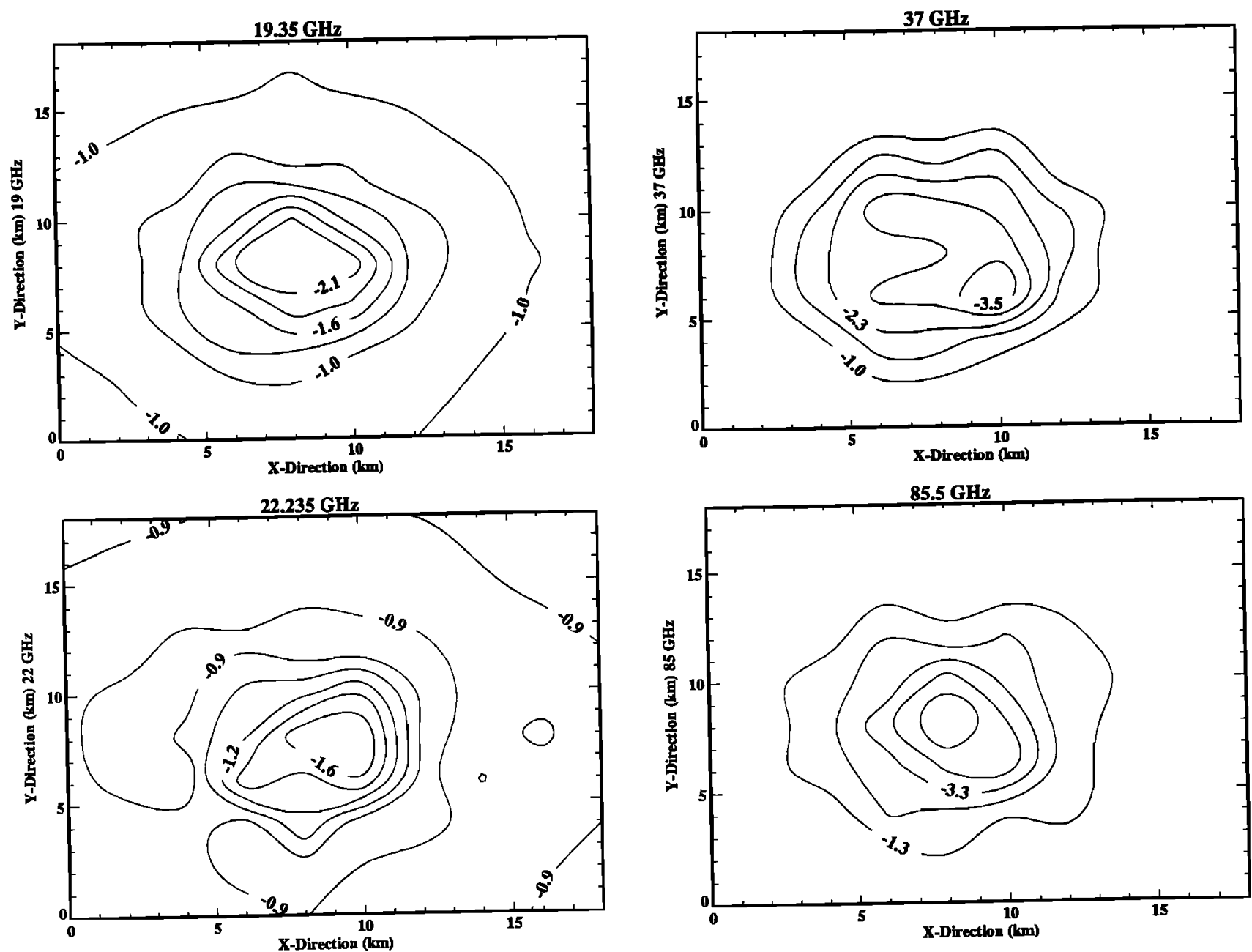

Figure 8a. Differences between the brightness temperatures calculated with the 3-D radiative transfer model and with the 1-D model at nadir. 

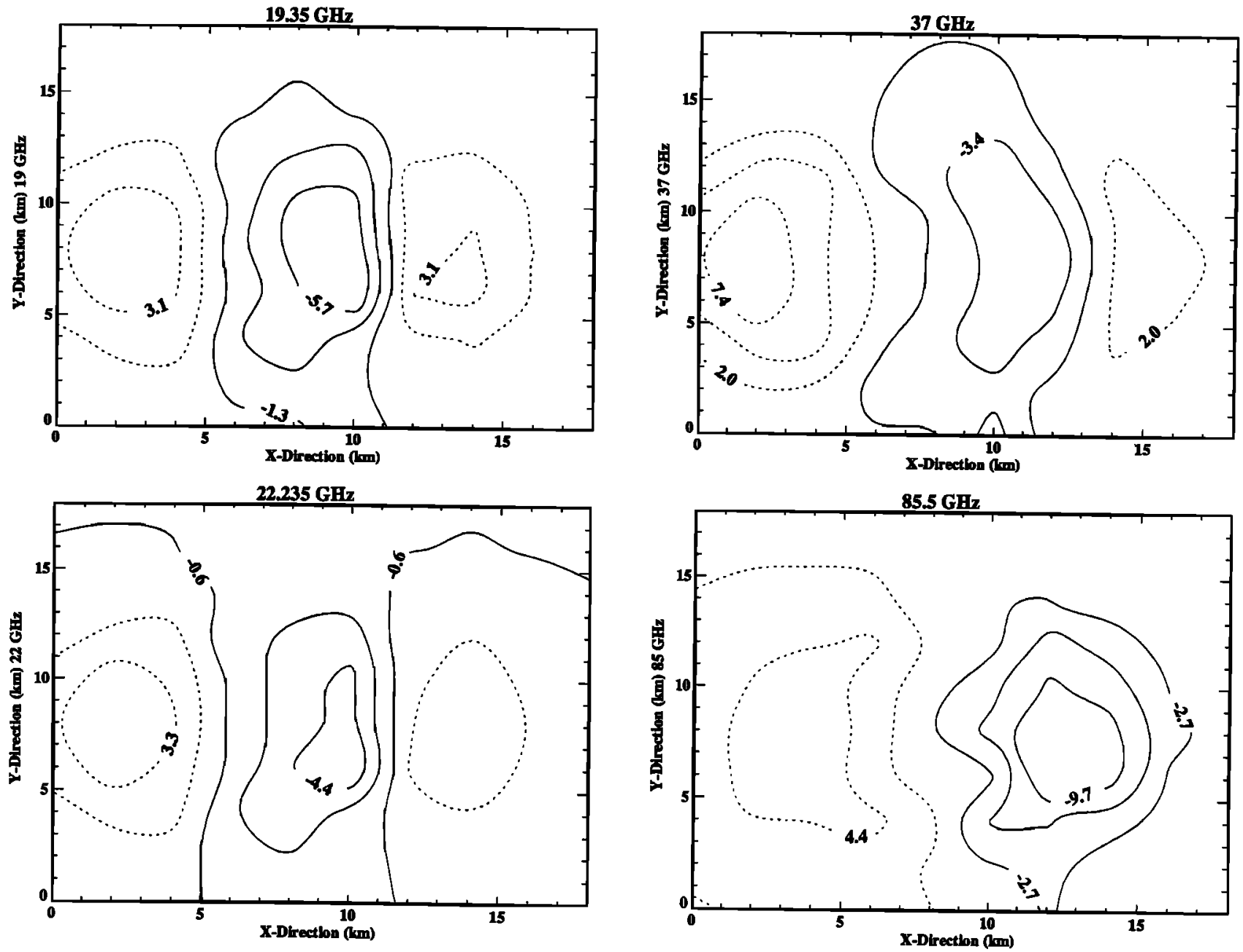

Figure 8b. Differences between the brightness temperatures calculated with the 3-D radiative transfer model and with the $1-\mathrm{D}$ model at viewing angle of $53^{\circ}$, vertical polarization.

\subsection{Three-Dimensional Dynamic Cloud Model}

The results with the box-type cloud may be partly unrealistic, for example, the sidewall effects may be exaggerated because of the abrupt changes from the homogeneous clouds to their environment. In order to investigate whether more realistic clouds show the same effects, we used results of the numerical cloud model GESIMA (Geesthacht simulation model of the atmosphere). GESIMA is a three-dimensional nonhydrostatic mesoscale model designed to study energy circulation, harmful gases transport, cloud dynamics, and radiation exchange between atmosphere and ocean [Kapitza and Eppel, 1992; Levkov et al., 1992; Eppel et al., 1995]. Model variables include profiles of the three-dimensional wind vector, temperature, humidity, cloud water and ice, rain, snow, and graupel.

The case studied here is based on one model run initialized with a radiosonde ascent on October 11, 1989, over the North Sea during the International Cirrus Experiment. The model area has horizontal dimensions of $20 \times 20 \mathrm{~km}$ with a resolution of $2 \times 2 \mathrm{~km}$, and extends vertically up to $10 \mathrm{~km}$ with 25 layers. Height cross sections through the center of the cloud for cloud and rain water and for total ice content are shown in Figure 7, also given is the rain rate at $50 \mathrm{~m}$ above ground.

Radiation transport simulations are carried out with the 3-D and the 1-D models. Extinction cross sections and single- scattering albedos are calculated using Mie theory, but the Rayleigh phase matrix is used to restrict the amount of computer time and memory. The 1-D simulations were obtained by treating each of the 100 columns of $2 \times 2 \times 10 \mathrm{~km}$ size as horizontally infinite and homogeneous. The nadir viewing brightness temperatures of the 3-D simulations are always lower than those of the 1-D model (Figure 8a) with maximum differences of over $5 \mathrm{~K}$ for $85.5 \mathrm{GHz}$. The results are consistent with the results obtained from the box-type cloud model. Therefore we can attribute these differences to the net leakage of radiation from the cloud sidewalls to the cloud-free environment.

For the viewing angle of $53^{\circ}$ (viewing from left to right in Figures $8 \mathrm{~b}$ and $8 \mathrm{c}$ is assumed) brightness temperatures from the 3-D simulations are higher than from the 1-D simulations in front of and behind the cloud. There are two exceptions: in the cloud center due to sidewall effects and for the brightness temperatures at $85.5 \mathrm{GHz}$ behind the cloud due to the strong scattering of the ice particles. As for the box-type cloud simulations, the pattern is almost symmetric for $19.35 \mathrm{GHz}$, while there exists a shift to larger differences in front of the cloud with increasing frequency (Figures $8 \mathrm{~b}$ and $8 \mathrm{c}$ ). We attribute the higher 3-D values in front of the cloud to the reflection effect and the higher values behind the cloud to the geometric ob- 

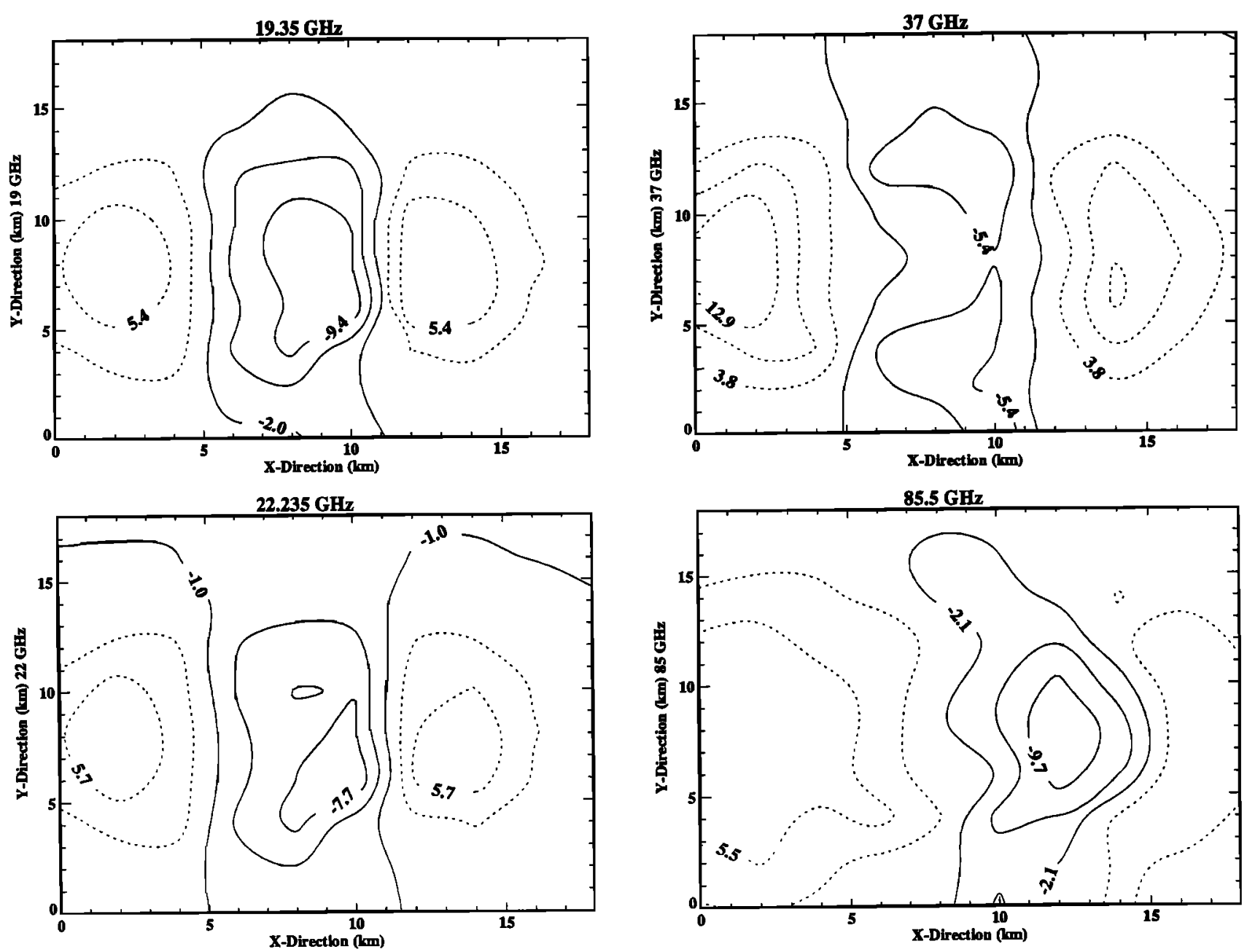

Figure 8c. Differences between the brightness temperatures calculated with the 3-D radiative transfer model and with the $1-\mathrm{D}$ model at viewing angle of $53^{\circ}$, horizontal polarization.

struction of the cloud-free water surface by the cloud. From Figures $8 \mathrm{~b}$ and $8 \mathrm{c}$ it follows, that the general structure of the differences (between 3-D and 1-D) are similar for both polarization states, but the absolute differences are always lower for the vertically polarized brightness temperatures. Since the vertically polarized brightness temperatures are in general larger than the the horizontally polarized brightness temperatures, we conclude, that the 3-D effects increase polarization differences in the center of the cloud and decrease polarization differences in front and behind the cloud.

\section{Conclusions}

Our study confirmed and explained the results of Kummerow and Weinman [1988] and Haferman et al. [1993], who calculated an underestimation of brightness temperatures by 1-D transfer models at off-nadir viewing angles and an overestimation at nadir, respectively. The nadir brightness temperature from the cloud from the finite cloud model is lower than the nadir brightness temperature from a horizontally homogeneous cloud model due to net leakage of radiation through the sidewalls of the cloud. This is what Haferman et al. [1993] found. The upwelling brightness temperature off nadir originates from the top and the side of the finite cloud. The up- welling brightness temperature from the side of the finite cloud can be larger than the brightness temperature from the top of the horizontally homogeneous cloud model due to the temperature increase from the cloud top to the surface. This explains the results of Kummerow and Weinman [1988].

Differences for $53^{\circ}$ viewing angle can approach $50 \mathrm{~K}$ for clear sky regions in the neighborhood of a rain cloud. The differences depend on frequency, viewing angle, and the optical properties of the cloud. For a finite rain cloud with a rainfall rate of $10 \mathrm{~mm} \mathrm{~h}^{-1}$ and a horizontal scale of $5 \times 5 \mathrm{~km}$ the calculated brightness temperature from 1-D model at 37 $\mathrm{GHz}$ is larger than the one from the 3-D model at nadir but smaller at the viewing angle of $53^{\circ}$. For more realistic clouds similar structures are obtained, but the effects are smaller.

A number of important conclusions can be drawn from our study. If we keep in mind, that real precipitation is of even smaller horizontal extent, than the cases modeled here, at least for convective rain, we can conclude that 3-D effects can lead to large deviations from the horizontally homogeneous (1-D) case.

These 3-D effects produce a highly nonlinear relationship between brightness temperatures and coverage of the rain column within the satellites' field of view; even if this coverage is 
known from an independent measurement, the linear decomposition into a rain and a nonrain column is not sufficient. This would take into account the so-called beam-filling effect, but the 3-D effects, which depend on the distribution of the rain cells in the field of view, will prevail. The 3-D effects also influence the polarization difference, which is an important variable in many rain retrieval algorithms.

For quantitive rain retrieval it will be necessary to parameterize the 3-D effects from the spatial distribution of rain cells in the field of view. To derive these parametrizations, much more must be known about the structure of precipitation on the small spatial scale. A combination of high-resolution remote sensing of precipitation structures from airplanes or from the surface should be combined with cloud models, into which these measurements can be assimilated. These data are an adequate data basis for 3-D radiative transfer modeling to work out parameterizations for 3-D effects in future rain retrieval algorithms.

However, there are also two good messages from our study: First, to a large extent the 3-D effects of radiative transfer can be accounted for by much less time-consuming modified 1-D modeling. Second, at least over the oceans, there is a tendency that radiation temperature depressions over cloud tops, due to net leakage of radiation into the surrounding cloud-free environment, is partially compensating for the increased radiation temperatures due to cloud sidewalls and their reflection from the water surface. For the cloud, simulated from the dynamic cloud model, the difference between the spatially averaged radiation temperatures from 1-D and 3-D modeling is only 1 to $3 \mathrm{~K}$. This is much smaller than obtained for the box-type cloud. However, additional cases simulated with spatial resolutions typical for precipitation clouds, the resolution in our example was only $1 \times 1 \mathrm{~km}$, have to be analyzed to arrive at a firm conclusion.

Acknowledgments. We wish to thank D. Ackermann for her assistance in providing data from the 3-D cloud model. We would like to thank the anonymous reviewers for their helpful comments. This work has been performed with funding support granted by Deutsche Forschungsgemeinschaft (DFG).

\section{References}

Adler, R. F., H.-Y. M. Yeh, N. Prasad, W.-K. Tao, and J. Simpson, Microwave simulations of a tropical rainfall system with a threedimensional cloud model, J. Appl. Meteorol., 30, 924-953, 1991.

Eppel, D. P., H. Kapitza, M. Claussen, D. Jacob, W. Koch, L. Levkov, H.-T. Mengelkamp, and N. Werrmann, The non-hydrostatic me- soscale model GESIMA, II, Parameterizations and Applications, Contrhb. Atmos. Phys., 68, 15-41, 1995.

Girens, K. M., A fast six-flux radiative transfer method for application in finite cloud models, Contrib. Atmos. Phys., 66, 73-87, 1993.

Haferman, J. L., W. F. Krajewski, T. F. Smith, and A. Sanchez, Radiative transfer for a three-dimensional raining cloud, Appl. Opt., 32, 2795-2802, 1993.

Kapitza, H., and D. P. Eppel, The non-hydrostatic mesoscale model GESIMA, I, Dynamical equations and tests, Contrib. Atmos. Phys., $65,129-146,1992$.

Kummerow, C., and J. A. Weinman, Determining microwave brightness temperatures from precipitating horizontally finite and vertically structured clouds, J. Geophys. Res., 93(4), 3720-3728, 1988.

Levkov, L., B. Rockel, H. Kapitza, and E. Raschke, 3-D numerical studies of cirrus and stratus clouds by their time and space evolution, Contrib. Atmos. Phys., 65, 35-58, 1992.

Liebe, H. J., An updated model for millimeter wave propagation in moist air, Radio Sci., 20, 1069-1089, 1985.

Liu, Q., An analytical solution of transmission and reflection operators for homogeneous atmospheres, Contrib. Atmos. Phys., 63, 128-133, 1990.

Liu, Q., C. Simmer, and E. Ruprecht, A general analytical expression of the radiation source function for emitting and scattering media within the matrix operator method, Contrib. Atmos. Phys., 64, 73-82, 1991.

McKee, T. B., and S. K. Cox, Scattering of visible radiation by finite clouds, J. Atmos. Sci., 31, 1885-1892, 1974.

Roberti, L., and C. Kummerow, A quantitative comparison between 3-D and plane parallel microwave radiative transfer codes applied to horizontally and vertically structured precipitating clouds, in Microwave Radiometry and Remote Sensing of the Environment, edited by D. Solimini, pp. 209-217, VSP, Utrecht, Netherlands, 1995.

Schmetz, J., On the parameterization of the radiative properties of broken clouds, Tellus Ser. A, 36, 417-432, 1984.

Spencer, R. W., A satellite passive $37-\mathrm{GHz}$ scattering-based method for measuring oceanic rain rates, J. Clim. Appl. Meteorol., 25, 754$766,1986$.

Stephens, G. L., Radıative transfer through arbitarily shaped optical media, J. Atmos. Scl., 45, 1818-1848, 1988.

Tsang, L., J. A. Kong, and R. T. Shin, Theory of Microwave Remote Sensing, 613 pp., John Wiley, New York, 1985.

Weinman, J. A., and R. Davies, Thermal microwave radiances from horizontally finite clouds of hydrometeors, J. Geophys. Res., 83(6), 3099-3107, 1978

Weinman, J. A., and P. J. Guetter, Determination of rainfall distributions from microwave radiation measured by the Nimbus 6 ESMR, J. Appl. Meteorol., 16, 437-442, 1977.

Wisler, M. M., and J. P. Hollinger, Estimation of marine environmental parameters using microwave radiometric remote sensing systems, NRL Memo. Rep., 3661, 1-27, 1977.

Q. Liu, C. Simmer, and E. Ruprecht, Institut fur Meereskunde, University of Kiel, Duesternbrooker Weg 20, Kiel 24105, Germany. (e-mail: qliu@ifm.uni-kiel.d400.de)

(Received November 14, 1994; revised October 25, 1995; accepted October 25, 1995.) 